\title{
Online Spectroscopic Study on the Positive and the Negative Electrolytes in Vanadium Redox Flow Batteries
}

\author{
Le Liu, ${ }^{1}$ Jingyu Xi, ${ }^{1,2}$ Zenghua $\mathrm{Wu}^{1}{ }^{1}$ Wenguang Zhang, \\ Haipeng Zhou, ${ }^{1}$ Weibin $\mathrm{Li}^{1},{ }^{1}$ and Yonghong $\mathrm{He}^{3}$ \\ ${ }^{1}$ Laboratory of Advanced Power Source, Graduate School at Shenzhen, Tsinghua University, Guangdong Province, \\ Shenzhen 518055, China \\ ${ }^{2}$ Key Laboratory of Thermal Management Engineering and Materials, Graduate School at Shenzhen, Tsinghua University, \\ Guangdong Province, Shenzhen 518055, China \\ ${ }^{3}$ Laboratory of Optical Imaging and Sensing, Graduate School at Shenzhen, Tsinghua University, Guangdong Province, \\ Shenzhen 518055, China \\ Correspondence should be addressed to Jingyu Xi; xijingyu@gmail.com
}

Received 4 July 2012; Revised 25 August 2012; Accepted 3 September 2012

Academic Editor: Djordje Mandrino

Copyright (C) 2013 Le Liu et al. This is an open access article distributed under the Creative Commons Attribution License, which permits unrestricted use, distribution, and reproduction in any medium, provided the original work is properly cited.

\begin{abstract}
Traditional spectroscopic analysis based on the Beer-Lambert law cannot analyze the analyte with high concentration and interference between different compositions, such as the electrolyte in vanadium redox flow batteries (VRBs). Here we propose a new method for online detection of such analytes. We demonstrate experimentally that, by comparing the transmittance spectrum of the analyte with the spectra in a preprepared database using our intensity-corrected correlation coefficient (ICCC) algorithm, parameters such as the state of charge (SOC) of both the positive and the negative electrolytes in the VRB can be online monitored. This method could monitor the level of the electrolytes imbalance in the VRB, which is useful for further rebalancing the electrolyte and restoring the capacity loss of the VRB. The method also has the potential to be used in the online detection of other chemical reactions, in which the chemical reagents have high concentration and interferences between different compositions.
\end{abstract}

\section{Introduction}

The technique of spectroscopy has been widely used in chemical composition analysis and the study of chemical reaction dynamics $[1,2]$. Traditional spectroscopic analysis is based on the Beer-Lambert law and has two requirements: (1) the concentration of the analyte is low; (2) there is no interference between different compositions of the analyte. However, analytes in many chemical and chemical engineering applications do not meet these two requirements and hence cannot be analyzed by the traditional Beer-Lambert law-based spectroscopic analysis.

The vanadium redox flow battery (VRB) is an energy storage technique that has caused wide concern in recent years [3, 4]. A VRB system includes one battery stack and two tanks. The positive and the negative electrolytes are stored in the two tanks separately and pumped into the stack, where the redox reactions take place. In the charge process, the tetravalent vanadium ions $\mathrm{V}(\mathrm{IV})$ in the positive electrolyte turn into pentavalent vanadium ions $\mathrm{V}(\mathrm{V})$, and the trivalent vanadium ions $\mathrm{V}(\mathrm{III})$ in the negative electrolyte turn into divalent vanadium ions $\mathrm{V}$ (II). In the discharge process, these reactions reverse. Ideally, the changes in concentration and the valence state of the positive and the negative electrolytes are the same. But in fact, a number of side reactions could lead to an imbalance between the positive and the negative electrolytes and a subsequent loss of capacity over extended charge-discharge cycling [5]. Thus a suitable electrolyte monitoring method is needed to detect the level of the imbalances.

Chemical methods such as potentiometric titration and chromatography have complicated steps and are not suitable for online detection $[6,7]$. Optical techniques such as spectroscopy fit the online detection demand. However, the concentration of the electrolyte in the VRB is too high (the typical value is close to $2 \mathrm{M}$ for total vanadium ions). The structure of the vanadium ions at such high concentration is 


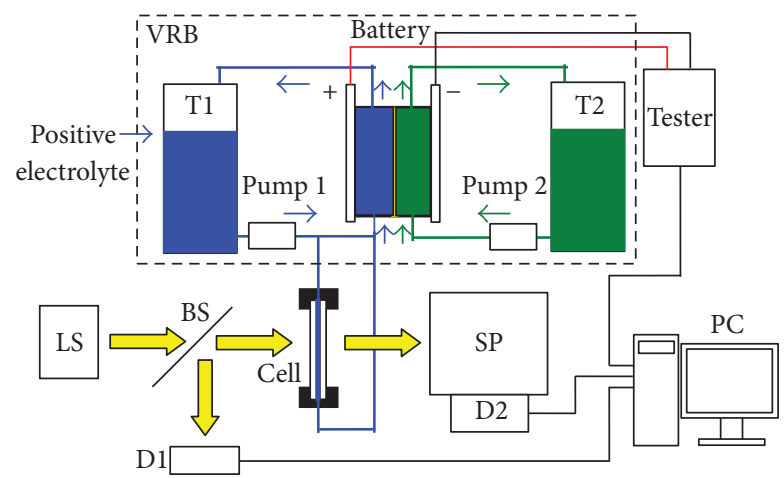

(a)

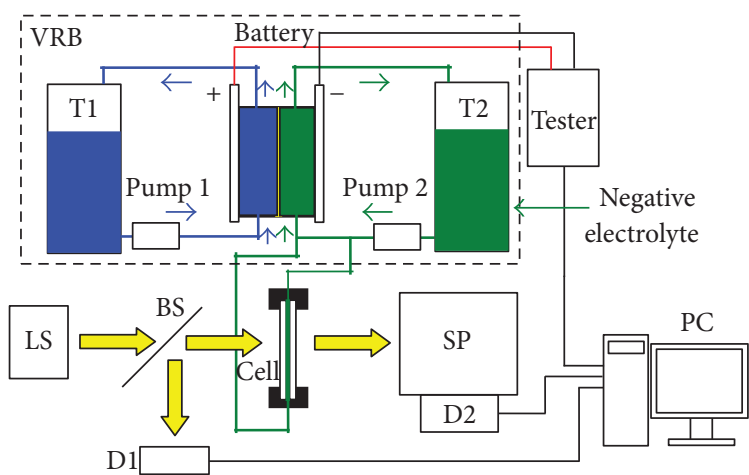

(b)

FIGURE 1: (a) The schematic of the spectroscopic online electrolyte analytical system when online detecting the positive electrolyte of a VRB. (b) The schematic of the same system when online detecting the negative electrolyte of a VRB. VRB: a vanadium redox flow battery; Battery: the battery stack of the VRB; T1 (T2): the positive (negative) electrolyte tank of the VRB; Pump 1 (2): the pump of the positive (negative) electrolyte; Tester: a charge-discharge tester; LS: a white light source; BS: a beam splitter; Cell: a 0.5 mm thick absorption flow cell; SP: a grating spectrometer; D1: a photo detector; D2: a cooled CCD camera; PC: a personal computer.

very complicated [8-10], and there are interferences between the vanadium ions with different valences. So the traditional Beer-Lambert law-based spectroscopic analysis cannot analyze the electrolyte in VRB [11].

In this paper, the variations of the transmission spectra of both the positive and the negative electrolytes in a VRB during the charging process are online studied by our homemade spectroscopic analytical system. A new method is proposed to analyze the spectra data of the analytes with high concentration and interferences between different compositions.

\section{System Setup}

The schematic of our spectroscopic online electrolyte analytical system is shown in Figure 1. Figures 1(a) and 1(b) are the schematics of the system when online detecting the positive and the negative electrolytes of a VRB, respectively.

The squared area in Figures 1(a) and 1(b) is a VRB system, which includes two electrolyte tanks (T1 in Figures 1(a) and 1(b), the positive electrolyte tank; T2 in Figures 1(a) and 1(b), the negative electrolyte tank), two pumps (Pump 1 and Pump 2 in Figures 1(a) and 1(b)), and a battery stack (Battery in Figures 1(a) and 1(b)). The positive and negative electrolytes are pumped from each tank into the battery stack, where the redox reactions take place, and then back into each tank. After pumped by Pump 1 (or Pump 2), a branch of the positive (or negative) electrolyte flows through a customized absorption flow cell (Cell in Figures 1(a) and 1(b)), which will be introduced below. The positive and negative electrodes of the battery stack are connected to a charge-discharge tester (Tester in Figures 1(a) and 1(b)), which is controlled by a personal computer (PC in Figures 1(a) and 1(b)).

Other part except the squared area in Figures 1(a) and 1 (b) is the spectroscopic analytical system. Parallel beam from a white light source (LS in Figures 1(a) and 1(b), a $150 \mathrm{~W}$ halogen lamp and the corresponding collimator) is split into two beams by a beam splitter (BS in Figures 1(a) and 1(b),
Thorlabs BS013). The reflected beam is detected by a photo detector (D1 in Figures 1(a) and 1(b), Texas Instruments OPT101), and the detection result is sent to the computer (PC in Figures 1(a) and 1(b)). The transmitted beam passes the customized absorption flow cell (Cell in Figures 1(a) and $1(\mathrm{~b})$ ), which allows the positive (or negative) electrolyte to flow between two glass windows and form a $0.5 \mathrm{~mm}$ thick uniform layer. The beam passing through the absorption cell goes into a grating spectrometer (SP in Figures 1(a) and 1(b), Acton Research SpectralPro 150). A cooled CCD camera (D2 in Figures 1(a) and 1(b)) captures the spectra of the light entering the spectrometer and sends the results to the computer (PC in Figures 1(a) and 1(b)).

The computer (PC in Figures 1(a) and 1(b)) controls the tester (Tester in Figures 1(a) and 1(b)) to charge and discharge the VRB stack (Battery in Figures 1(a) and 1(b)). Meanwhile, from the fluctuation of the light source detected by the detector (D1 in Figures 1(a) and 1(b)) and the spectrum of the electrolyte detected by the CCD (D2 in Figures 1(a) and 1(b)), we can obtain the normalized transmission spectrum of the $0.5 \mathrm{~mm}$ thick positive (or negative) electrolyte in real time.

\section{Experimental}

3.1. Spectra of the Electrolytes in VRB. The electrolyte with 2.0 M V(IV) and 3.0 $\mathrm{M} \mathrm{H}_{2} \mathrm{SO}_{4}$ is prepared as the initial positive electrolyte; the electrolyte with $2.0 \mathrm{M} \mathrm{V}$ (III) and $3.0 \mathrm{M}$ $\mathrm{H}_{2} \mathrm{SO}_{4}$ is prepared as the initial negative electrolyte. When detecting the positive electrolyte, the volume of the negative electrolyte is set $25 \%$ larger than the volume of the positive electrolyte, so that all V(IV) ions in the positive electrolyte can be fully charged into $\mathrm{V}(\mathrm{V})$ ions. When detecting the negative electrolyte, the volume of the positive electrolyte is set $25 \%$ larger than the volume of the negative electrolyte, so that all V(III) ions in the negative electrolyte can be fully charged into V(II) ions. The state of charge (SOC) can be used to quantify the charge level of the battery. For example, for 


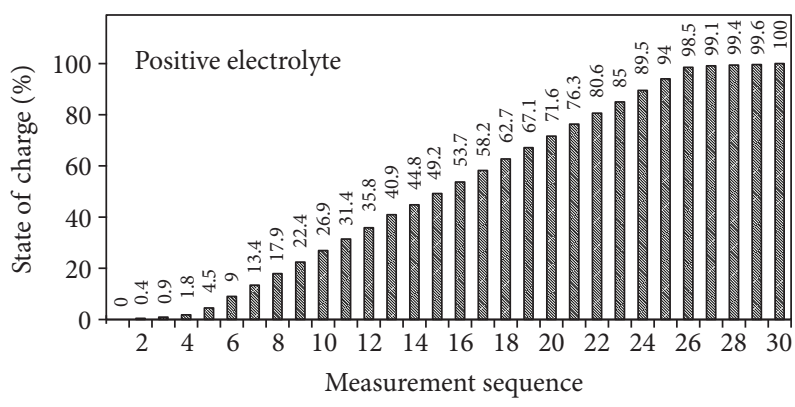

(a)

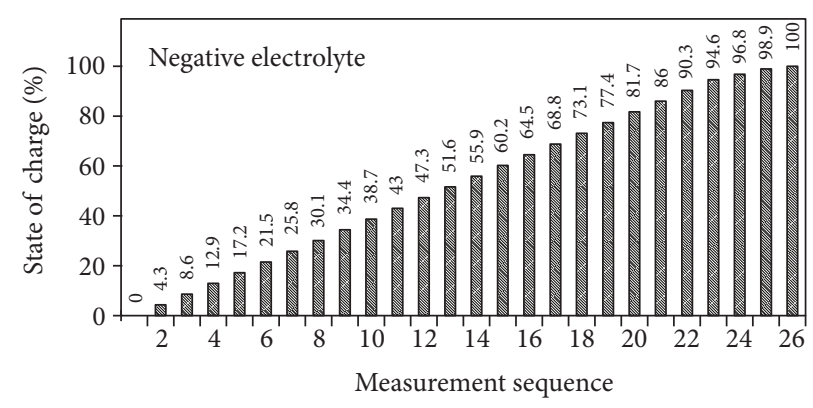

(b)

FIGURE 2: (a) The state of charge (SOC) of each measurement for the positive electrolyte. (b) The state of charge (SOC) of each measurement for the negative electrolyte.

the positive electrolyte, the SOC is $0 \%$ when all vanadium ions are $\mathrm{V}(\mathrm{IV})$, the SOC is $100 \%$ when all vanadium ions become $\mathrm{V}(\mathrm{V})$; for the negative electrolyte, the SOC is $0 \%$ when all vanadium ions are $\mathrm{V}(\mathrm{III})$, the SOC is $100 \%$ when all vanadium ions become $\mathrm{V}(\mathrm{II})$.

The computer controls the tester to charge the battery; meantime the system in Figure 1 measures the transmittance spectra of the positive (or negative) electrolyte in different SOCs. We take 30 measurements for the positive electrolyte and 26 measurements for the negative electrolyte from SOC of $0 \%$ to SOC of $100 \%$. The SOCs of each measurement are shown in Figures 2(a) and 2(b).

The measured 30 normalized transmittance spectra of the positive electrolyte are shown in Figures 3(a)-3(d). We can see that only measurements $1-7$ and 25-30 are shown in Figure 3(a); that is because other measured spectra are too small to be seen. The marked area in Figure 3(a) is enlarged and shown in Figure 3(b) with measurements 7-11 and 23-25. Again the marked area in Figure 3(b) is further enlarged and shown in Figure 3(c) with measurements 11-15 and 20-23. Once more the marked area in Figure $3(c)$ is further enlarged and shown in Figure 3(d) with measurements 15-20. It is shown from Figure 3(a) to Figure $3(\mathrm{~d})$ that the pure V(IV) (measurement 1, SOC 0\%) and pure $\mathrm{V}(\mathrm{V})$ (measurement 30, SOC 100\%) have the similar transmittance intensity (around 0.8). With the measurements continued from measurement 1 (SOC $0 \%$ ), the intensity of the transmittance spectra drops quickly. At the 18th measurement (SOC 62.7\%), the intensity of the transmittance spectra reaches the minimum (approximate $1.1 \times 10^{-3}$ ). Then with the measurements continued from measurement 18 , the intensity of the transmittance spectra rises quickly and finally resumes to around 0.8 at the 30th measurement (SOC 100\%). We can also conclude from Figures 3(a)-3(d) that, in addition to the dramatic changes in the intensity of the transmittance spectra, the shape of the transmittance spectra also changes and the peak of the spectra moves to the longer wavelength.

The average transmittances of each measurement in Figure 3 are shown in Figure 4(a), and the peak wavelengths within the measured spectral range of each measurement in Figure 3 are shown in Figure 4(b). It can be seen from Figure 4(a) that the average intensity of the transmittance spectra of the positive electrolyte drops then rises with measurements continued (SOC increases) and the 18th spectrum has the minimal intensity. It can be concluded from Figure 4(b) that the peak wavelength of the transmittance spectra of the positive electrolyte increases with measurements continued (SOC increases). These results agree with the above results in Figure 3. Because the intensity of the transmittance spectral of the positive electrolyte is too small in quite a range of SOC, using the absorption spectra as the detection result will lead to a bad signal-to-noise ratio (SNR). Hence, in this study, we use the transmittance spectra instead of the absorption spectral as the detection results.

The measured 26 normalized transmittance spectra of the negative electrolyte are shown in Figures 5(a) and 5(b). It is shown from Figure 5(a) that the pure V(III) (measurement 1 , SOC $0 \%$ ) has a peak near $500 \mathrm{~nm}$ with the peak intensity between 0.5 and 0.6 . With the measurements continued (SOC increases), the peak of the spectrum move to the shorter wavelength and the peak intensity decreases. At the 8th measurement (SOC 30.1\%), the intensity of the transmittance spectra reaches the minimum (approximate 0.45 ). We can see from Figure 5(b) that with the measurements continued from measurement 8 , the peak of the spectrum continues to move to the shorter wavelength and the peak intensity begins to increase. At the 26th measurement (pure V(II), SOC 100\%), the peak wavelength is around $430 \mathrm{~nm}$ and the peak intensity reaches near 0.9. From Figure 5, we can conclude that the intensity of the spectra of the negative electrolyte drops then rises with measurements continued (SOC increases), and this change of intensity is much smaller than the spectra of the positive electrolyte in Figure 3. It can also be seen that, at certain wavelength, for example, $430 \mathrm{~nm}$, the transmittance increases with the measurements (SOC) in the whole process.

The transmittances of each measurement at $430 \mathrm{~nm}$ in Figure 5 are plotted in Figure 6(a), and the peak wavelengths within the measured spectral range of each measurement in Figure 5 are shown in Figure 6(b). It can be seen from Figure 6 (a) that the transmittance at $430 \mathrm{~nm}$ of the negative electrolyte increases exponentially with the SOC. This is because $430 \mathrm{~nm}$ is near the absorption peak (transmittance bottom) of V(III) and is about the absorption bottom (transmittance peak) of V(II). It can be concluded from Figure 6(b) that the 


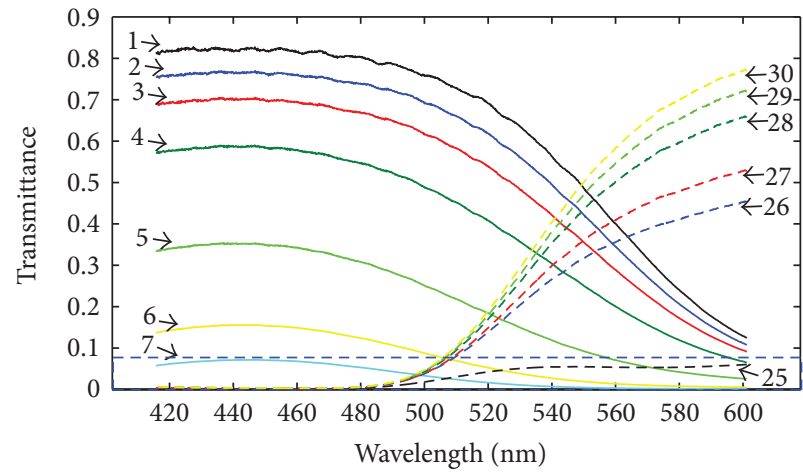

(a)

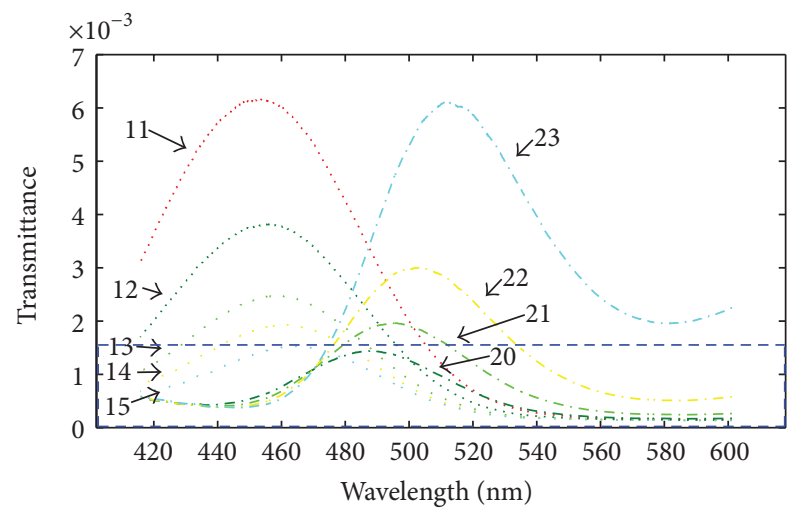

(c)

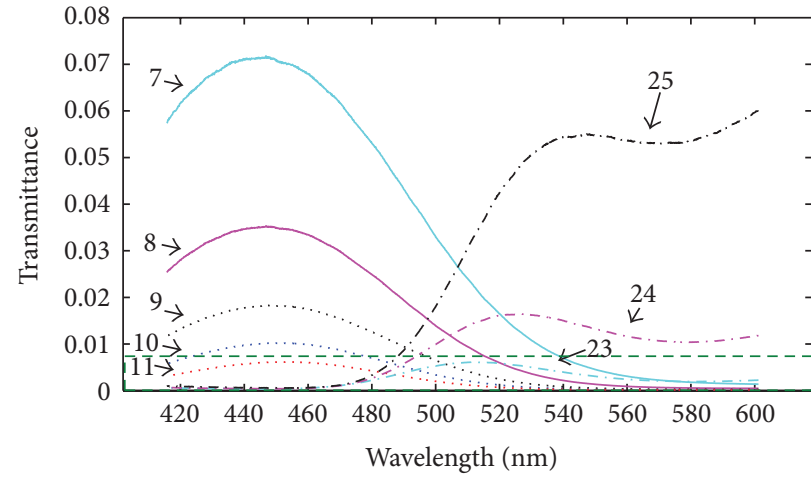

(b)

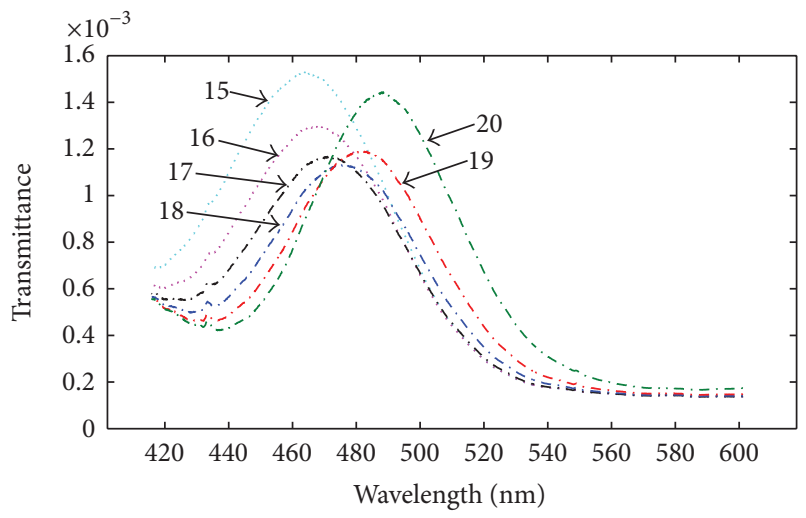

(d)

FIGURE 3: The normalized transmittance spectra of the 30 measurements for the positive electrolyte in Figure 2(a). The marked area in (a) is enlarged and shown in (b); the marked area in (b) is enlarged and shown in (c); the marked area in (c) is enlarged and shown in (d).

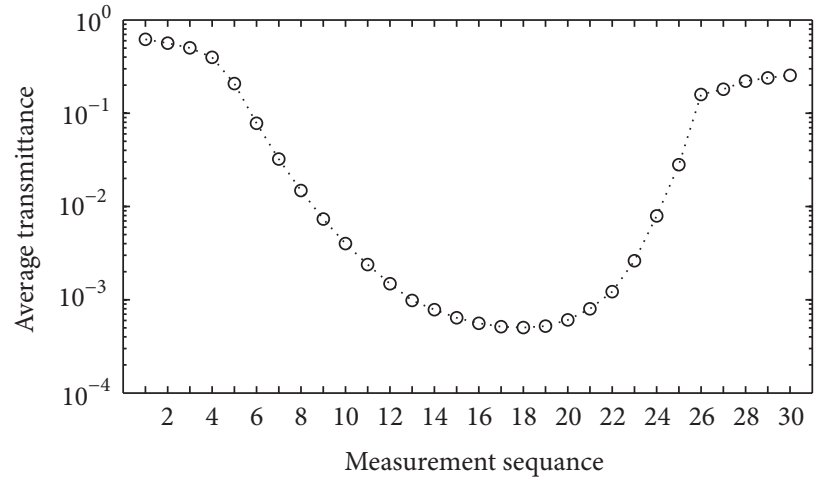

(a)

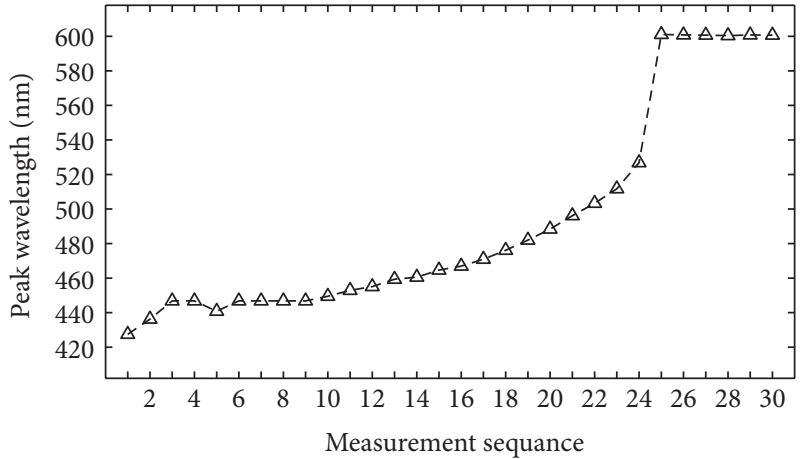

(b)

FIGURE 4: (a) The mean of the spectra of the 30 measurements for the positive electrolyte in Figure 3; (b) the peak wavelength within the measurement range $(420 \mathrm{~nm}-600 \mathrm{~nm})$ of the spectra of the 30 measurements for the positive electrolyte in Figure 3. 

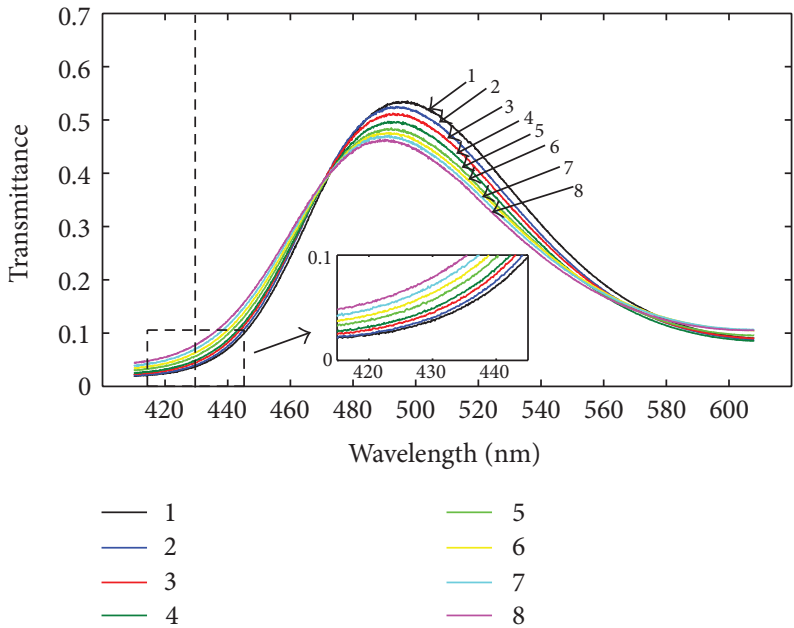

(a)

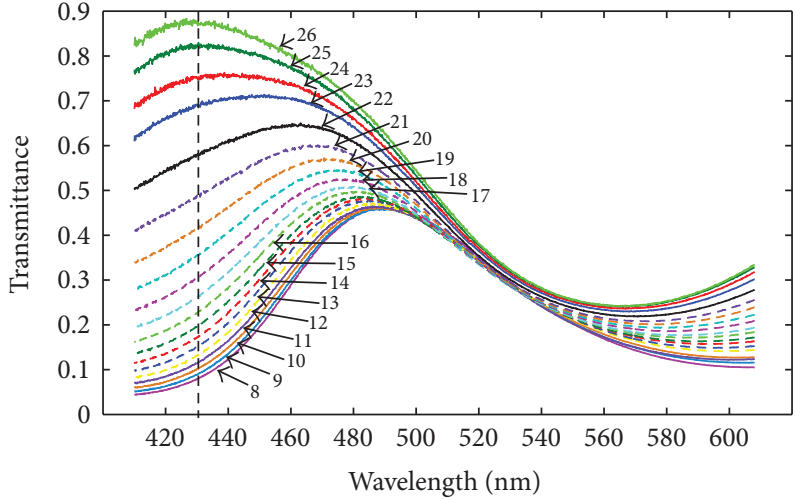

$\begin{array}{ll}-8 & --18 \\ -9 & --19 \\ -10 & --20 \\ -11 & --21 \\ --12 & -22 \\ --13 & -23 \\ ---14 & -24 \\ ---16 & -25 \\ - & -26\end{array}$

(b)

FIGURE 5: The normalized transmittance spectra of the 26 measurements for the negative electrolyte in Figure 2(b). The marked area in (a) is enlarged and shown in the bottom of (a).

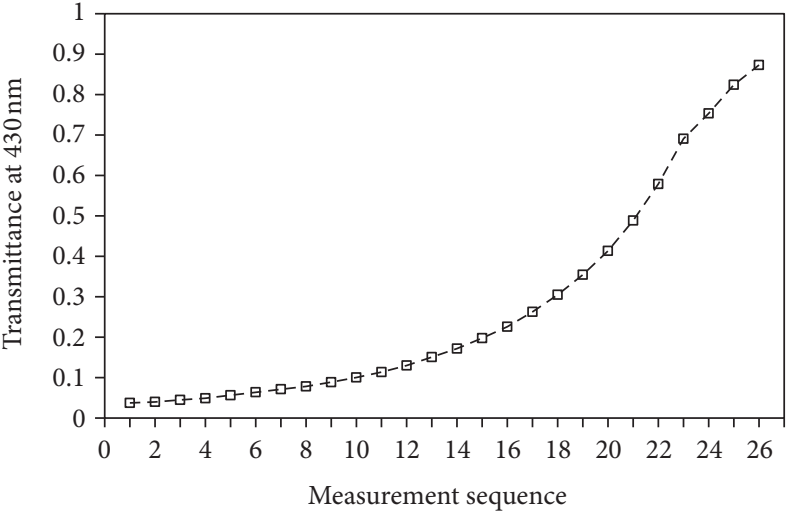

(a)

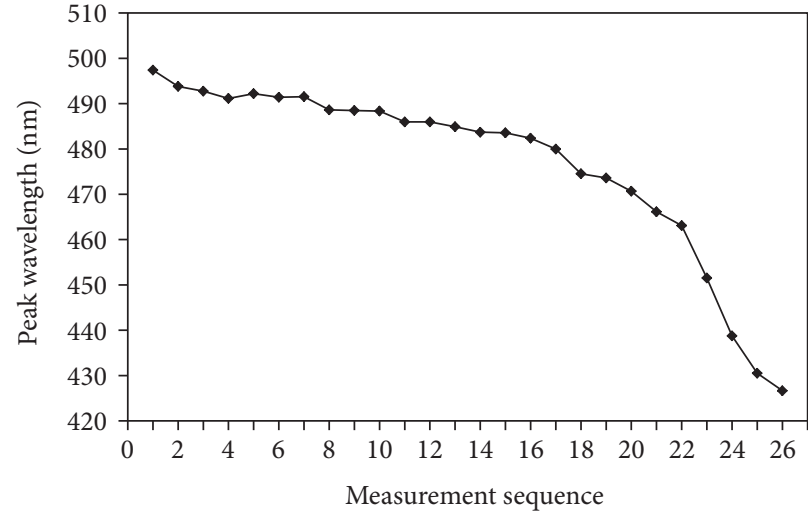

(b)

FiguRE 6: (a) The transmittances at $430 \mathrm{~nm}$ of 26 measurements for the negative electrolyte in Figure 5; (b) the peak wavelength within the measurement range $(420 \mathrm{~nm}-600 \mathrm{~nm})$ of the spectra of the 26 measurements for the negative electrolyte in Figure 5.

peak wavelength of the transmittance spectra of the negative electrolyte decreases with measurements continued (SOC increases). These results agree with the above results in Figure 5.

3.2. Methods and Algorithm for Spectroscopic Analysis. We can conclude from Figures 5 and 6 that the transmittance spectra of the negative electrolyte with different SOCs obey the Beer-Lambert law. However, we can see from Figures 3 and 4 that, when the SOC increases, the transmittance spectra of the positive electrolyte changes dramatically both in intensities and in shapes. Thus, traditional Beer-Lambert law-based spectroscopic analysis cannot analyze the positive electrolyte in the VRB. Hence, new spectral data analysis method is needed.

In this paper, we propose a new method for analyzing the spectral data of the electrolyte in the VRB.

(1) The positive (or negative) electrolytes with different parameters in the VRB during charging for the first time are used as the standard samples. The parameters of the electrolyte include the type of the electrolyte (positive of negative), the SOC, the total vanadium concentration, and the $\mathrm{H}_{2} \mathrm{SO}_{4}$ concentration. For 


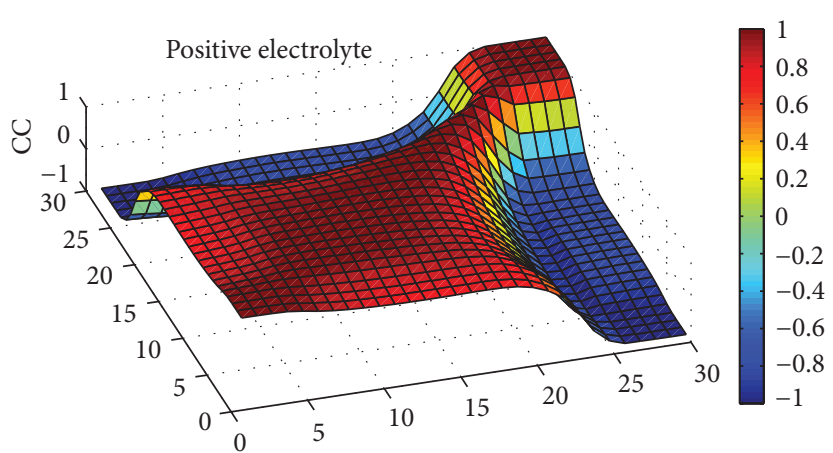

(a)

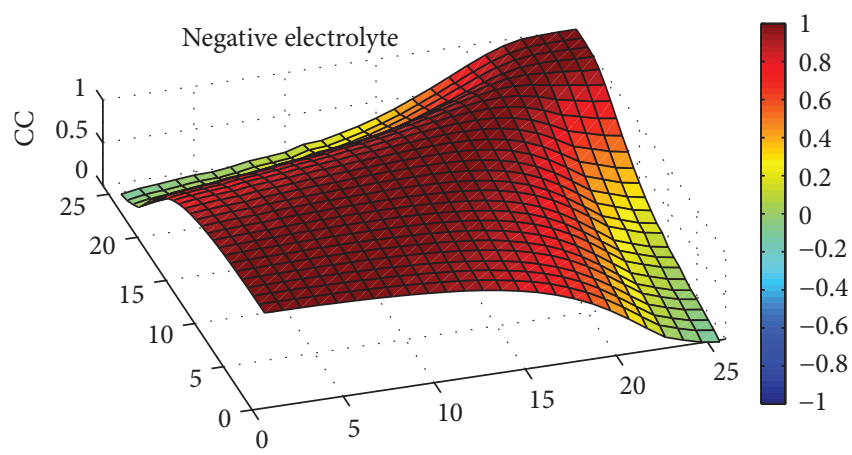

(c)

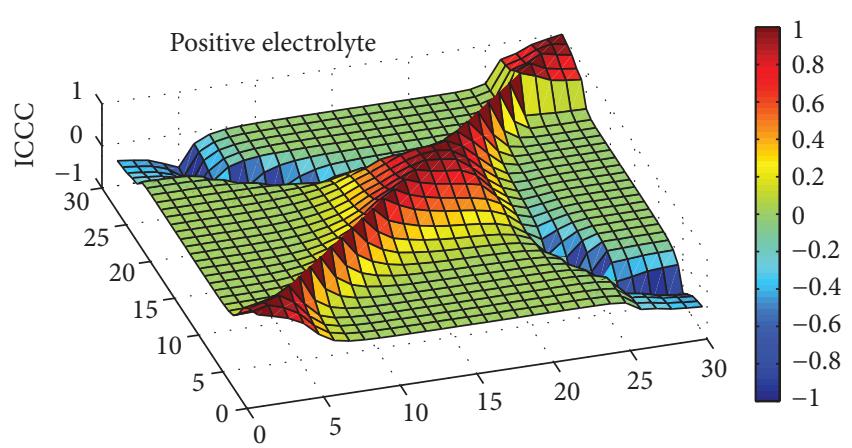

(b)

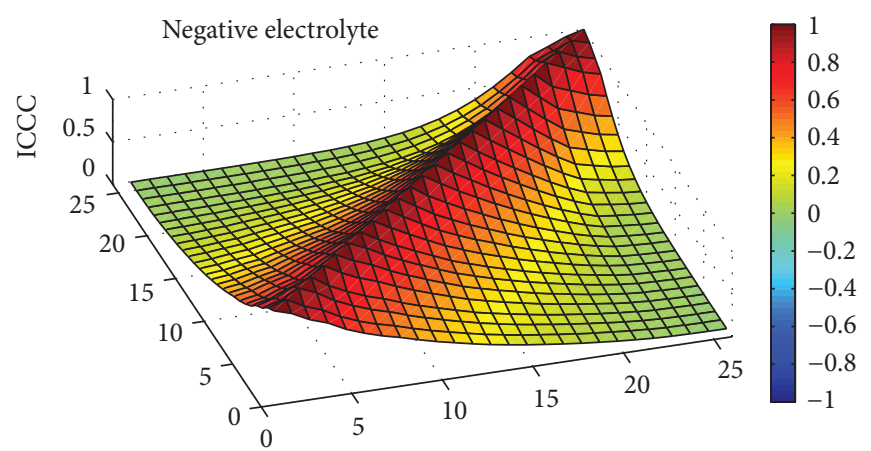

(d)

FIGURE 7: (a) The correlation coefficients (CCs) of the spectra of the 30 measurements for the positive electrolyte in Figure 3; (b) the intensitycorrected correlation coefficients (ICCCs) of the spectra of the 30 measurements for the positive electrolyte in Figure 3; (c) the correlation coefficients (CCs) of the spectra of the 26 measurements for the negative electrolyte in Figure 5; (d) the intensity-corrected correlation coefficients (ICCCs) of the spectra of the 26 measurements for the negative electrolyte in Figure 5.

example, the spectral data in Figure 3 are such a group of standard samples of the positive electrolyte with the total vanadium concentration of $2.0 \mathrm{M}$ and the $\mathrm{H}_{2} \mathrm{SO}_{4}$ concentration of $3.0 \mathrm{M}$. The same spectroscopic analytical system as mentioned in Figure 1 is used to detect the normalized transmittance spectra of all standard samples and save these spectral data and the corresponding parameters of the electrolytes as a database.

(2) During online detection of a VRB with the same system, the detected normalized transmittance spectrum of the positive electrolyte in the VRB is compared with all the spectral data in the database. Certain algorithm, which we will discuss in the following, is used to determine the most similar spectrum in the database, and the corresponding parameters (e.g., SOC) of this data are considered as the parameters of the detected electrolyte in the VRB.

The algorithm of correlation is often used as a tool for characterizing the similarity of two sets of data [12]. The correlation coefficient (CC) of two $n$-order vectors $X(i)$ and $Y(i)$ is defined by

$$
\mathrm{CC}=\frac{\sum_{i=1}^{n}\left(X_{i}-\bar{X}\right)\left(Y_{i}-\bar{Y}\right)}{\sqrt{\sum_{i=1}^{n}\left(X_{i}-\bar{X}\right)^{2}} \sqrt{\sum_{i=1}^{n}\left(Y_{i}-\bar{Y}\right)^{2}}},
$$

where $\bar{X}$ and $\bar{Y}$ are the mean values of vectors $X(i)$ and $Y(i)$, respectively.

The CCs of the 30 spectral data of the positive electrolyte in Figure 3 are shown in Figure 7(a), and the CCs of the 26 spectral data of the negative electrolyte in Figure 5 are shown in Figure 7(c). The diagonal elements in Figures 7(a) and 7 (c) are the self-correlation coefficients (self-CCs) of each spectrum, the nondiagonal elements are the cross-correlation coefficient (cross-CC) of two different spectra. The value of $\mathrm{CC}$ is between 1 and -1 . The larger the $\mathrm{CC}$ is, the more similar the two spectra are. In the ideal situation, where all spectra are able to distinguish with other spectra, the diagonal elements should be 1 and the nondiagonal elements should be much less than 1. However, we can see from Figures 7(a) and 7(c) that some elements near the diagonal elements are too close to 
1, that it is difficult to distinguish some of the adjacent spectra by the CC algorithm.

The reason CC cannot be used to distinguish the spectra in Figure 3 and Figure 5 is that the correlation coefficient algorithm is sensitive to the shape but not the intensity of the data. But the spectra in Figures 3 and 5 change both in intensities and in shapes. Therefore, we propose an intensitycorrected correlation coefficient (ICCC) to compare the spectra. The ICCC for the positive electrolyte is defined by

$$
\mathrm{ICCC}=\mathrm{CC} \cdot \frac{\operatorname{Min}(\bar{X}, \bar{Y})}{\operatorname{Max}(\bar{X}, \bar{Y})},
$$

where $\operatorname{Min}(A, B)$ is the smaller one in $A$ and $B$ and $\operatorname{Max}(A$, $B$ ) is the larger one in $A$ and $B$. The ICCC for the negative electrolyte is defined by

$$
\operatorname{ICCC}=\mathrm{CC} \cdot \frac{\operatorname{Min}\left(X_{430}, Y_{430}\right)}{\operatorname{Max}\left(X_{430}, Y_{430}\right)},
$$

where $X_{430}$ and $Y_{430}$ are the elements at $430 \mathrm{~nm}$ of vectors $X(i)$ and $Y(i)$, respectively.

The ICCCs of the 30 spectral data of the positive electrolyte in Figure 3 are shown in Figure 7(b), and the ICCCs of the 26 spectral data of the negative electrolyte in Figure 5 are shown in Figure 7(d). The same as Figures 7(a) and $7(\mathrm{c})$, the diagonal elements are the intensity-corrected self-correlation coefficients (self-ICCCs) of each spectrum, the nondiagonal elements are the intensity-corrected crosscorrelation coefficient (cross-ICCC) of two different spectra. The value of ICCC is also between 1 and -1 . The larger the ICCC is, the more similar the two spectra are. We can conclude from Figures 7(b) and 7(d) that the diagonal elements are 1, and the value of the elements drops quickly away from the diagonal. In fact, the largest value of the nondiagonal elements is 0.96 .

It should be noted that, unlike the positive electrolyte, the transmittance spectra of the negative electrolyte obey the Beer-Lambert law (Figure 5). So we can detect the SOC of the negative electrolyte by the transmittance at one wavelength (e.g., $430 \mathrm{~nm}$, Figure 6(a)). However, compared with this traditional one wavelength analyzing method, our ICCC-based method makes use of the entire spectral data, ensures the accuracy of detection, and has the capability to distinguish between varieties of parameters besides the SOC.

3.3. Detection Repeatability and Resolution. To test the detection repeatability of our method, we detect the spectra of the electrolyte ten times during each measurement corresponding different SOC in Figures 2(a) and 2(b). The resulting ten spectra in the same measurement are then used to calculate the ICCC of each other. The minimum of the ICCCs from each measurement is shown in Figure 8. It can be concluded from Figure 8 that all the ICCCs are larger than 0.99, which shows the repeatability of our spectral analytical method.

The ICCCs in Figure 8 characterize the uncertainty of the detection based on our spectroscopy detection system and our spectra analytical algorithm. The ICCCs in Figure 8 (above 0.99) are larger than the nondiagonal elements in

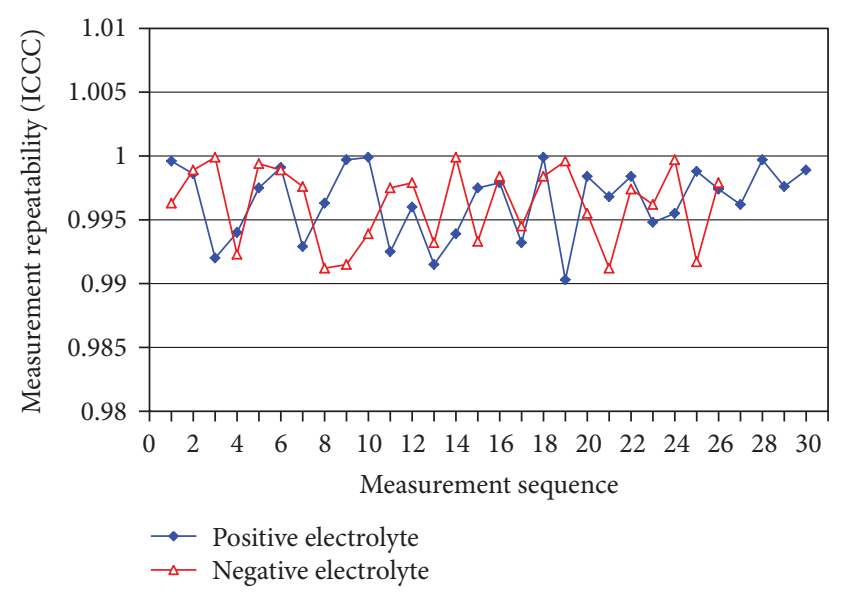

Figure 8: The repeatability of the measurements. Each measurement in Figures 2(a) and 2(b) has ten detections, resulting in ten spectra. Ten spectra in the same measurement are used to calculate the intensity-corrected correlation coefficients (ICCCs) of each other. The minimum of the ICCCs from each measurement for both the positive and the negative electrolytes is plotted here.

Figures 7(b) and 7(d) (below 0.96), which indicates that our system and our algorithm based on ICCC can distinguish all the spectra in the measurements in Figures 3 and 5. Therefore, the resolution of our system is better than the smallest SOC differences between each two measurements in Figure 2, which is $0.3 \%$ of SOC for the positive electrolyte and $2.1 \%$ of SOC for the negative electrolyte. This resolution is enough for monitoring the imbalance of the electrolyte in a VRB.

We have demonstrated that our system and method can monitor the SOC of both the positive and the negative electrolytes in the VRB. With expanded database including spectra data with more parameters, our method can be used to online determine more parameters of the electrolyte in the $\mathrm{VRB}$, including the SOC, the total vanadium concentration, the and $\mathrm{H}_{2} \mathrm{SO}_{4}$ concentration.

\section{Conclusion}

A homemade spectroscopic system is built to study the variation of the transmission spectra of both the positive and the negative electrolytes in a VRB during the charging process. A new spectroscopic method is proposed to online analyze the electrolytes in a VRB, which cannot be analyzed by the traditional Beer-Lambert law-based spectroscopic method because of their high concentration and interferences between the vanadium ions with different valences. We demonstrate that, by comparing the normalized transmittance spectrum of the analyte with the spectral data in the database using our ICCC algorithm, the parameters (including the SOC, the total vanadium concentration, and the $\mathrm{H}_{2} \mathrm{SO}_{4}$ concentration) of the electrolyte in the VRB can be monitored. Our method could monitor the level of the electrolytes imbalance in the VRB, which is useful for further rebalancing the electrolyte and restoring the capacity loss of the VRB. This method also has the potential to be used in 
the online detection of other chemical reactions, in which the chemical reagents have high concentration and interferences between different compositions.

\section{Acknowledgments}

This research was made possible with the financial support from the National Natural Science Foundation of China (20973099, 81171375, and 61040067), Program for New Century Excellent Talents in University, Shenzhen Science Fund for Distinguished Young Scholars (2010, 2011), and the Basic Research Program of Shenzhen City (JC201005310703A, JC201005310712A, JC200903180530A, and CXB201005250040A).

\section{References}

[1] D. J. Leggett, "Numerical analysis of multicomponent spectra," Analytical Chemistry, vol. 49, no. 2, pp. 276-281, 1977.

[2] X. Chen, J. M. Ma, K. L. Yong, J. C. Lv, and X. B. Zhang, "Fluorescence study on the interaction of human serum albumin with loureirin B," Spectroscopy, vol. 24, no. 5, pp. 547-557, 2010.

[3] M. Skyllas-Kazacos, M. Rychcik, R. G. Robins, A. G. Fane, and M. A. Green, "New all-vanadium redox flow cell," Journal of the Electrochemical Society, vol. 133, no. 5, pp. 1057-1058, 1986.

[4] A. Z. Weber, M. M. Mench, J. P. Meyers, and etal, "Redox flow batteries: a review," Journal of Applied Electrochemistry, vol. 41, pp. 1137-1164, 2011.

[5] T. Ao, B. Jie, and M. Skyllas-Kazacos, "Dynamic modelling of the effects of ion diffusion and side reactions on the capacity loss for vanadium redox flow battery," Journal of Power Sources, vol. 24, pp. 10737-10747, 2011.

[6] B. Tian, C. W. Yan, Q. Qu et al., "Potentiometric titration analysis of electrolyte of vanadium battery," Battery Bimonthly, vol. 33, pp. 261-263, 2003.

[7] L. Tian, L. Liu, L. Chen, N. Lu, and H. Xu, "Electrochemical determination of iodide on a vanadium oxide-polypropylene carbonate coated glassy carbon electrode," Talanta, vol. 66, no. 1, pp. 130-135, 2005.

[8] M. Vijayakumar, L. Y. Li, Z. Nie et al., "Structure and stability of hexa-aqua V(III) cations in vanadium redox flow battery electrolytes," Physical Chemistry Chemical Physics, vol. 14, pp. 10233-10242, 2012.

[9] M. Vijayakumar, L. Li, G. Graff et al., "Towards understanding the poor thermal stability of $\mathrm{V}^{5+}$ electrolyte solution in vanadium redox flow batteries," Journal of Power Sources, vol. 196, no. 7, pp. 3669-3672, 2011.

[10] M. Vijayakumar, S. D. Burton, C. Huang et al., "Nuclear magnetic resonance studies on vanadium(IV) electrolyte solutions for vanadium redox flow battery," Journal of Power Sources, vol. 195, no. 22, pp. 7709-7717, 2010.

[11] M. Skyllas-Kazacos and M. Kazacos, "State of charge monitoring methods for vanadium redox flow battery control," Journal of Power Sources, vol. 196, no. 20, pp. 8822-8827, 2011.

[12] http://en.wikipedia.org/wiki/Pearson_product-moment_correlation_coefficient. 

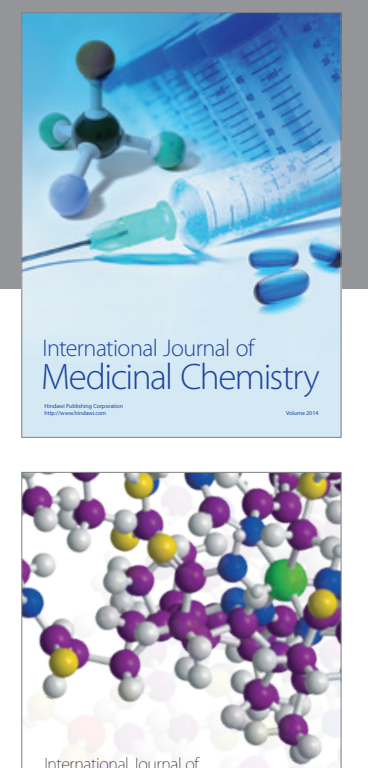

\section{Carbohydrate} Chemistry

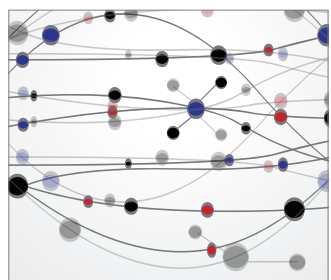

The Scientific World Journal
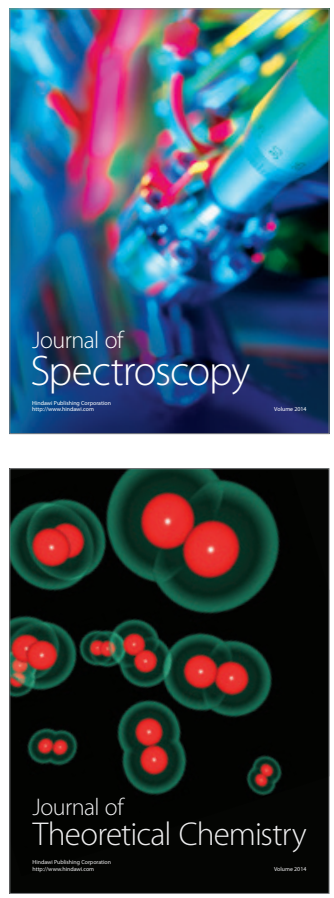
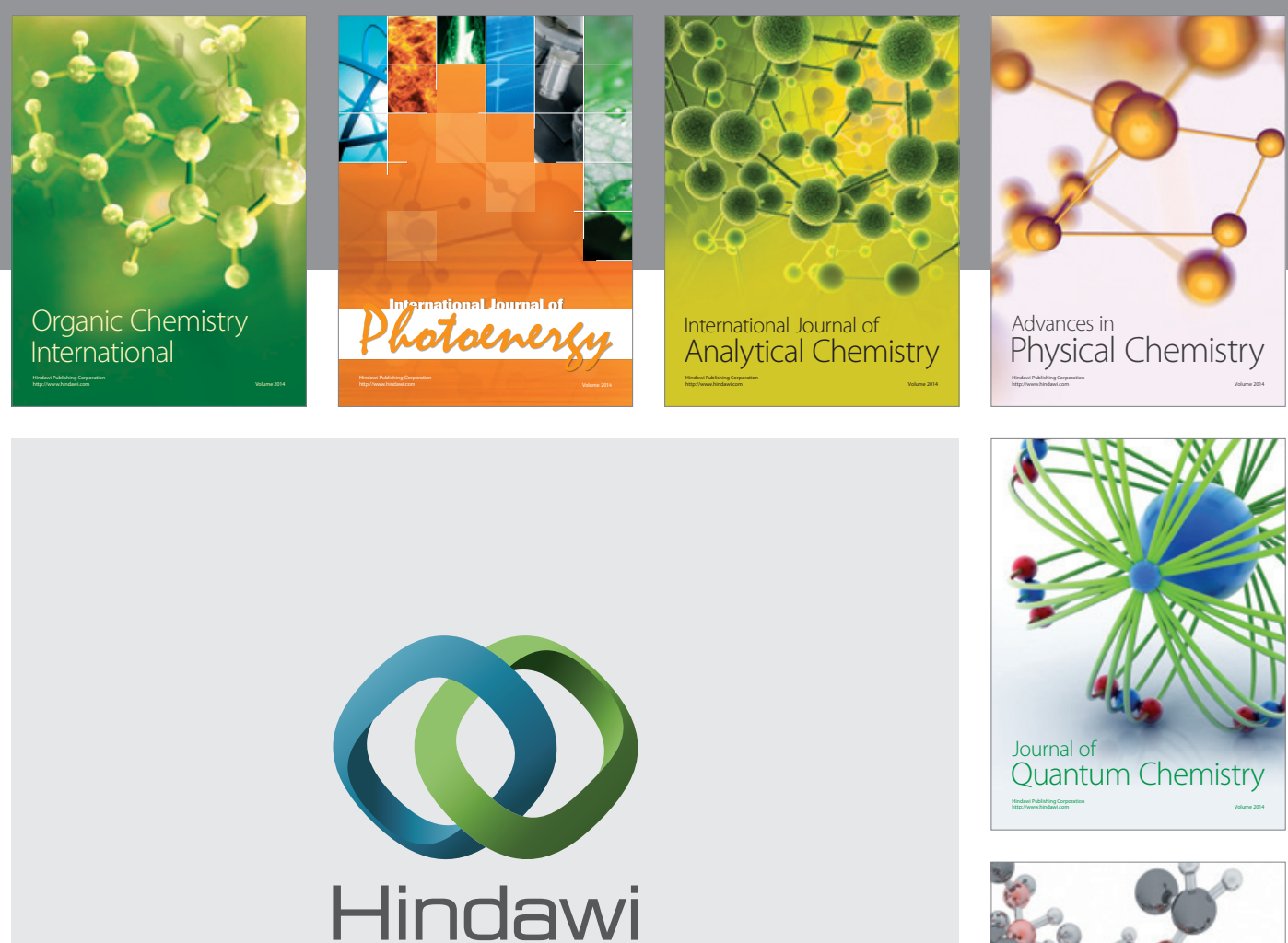

Submit your manuscripts at

http://www.hindawi.com

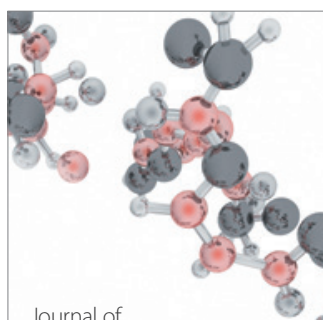

Analytical Methods

in Chemistry

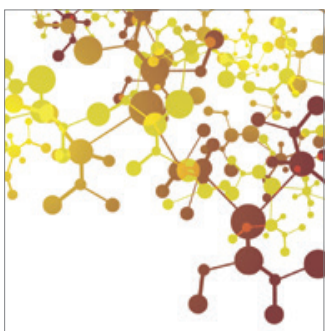

Journal of

Applied Chemistry

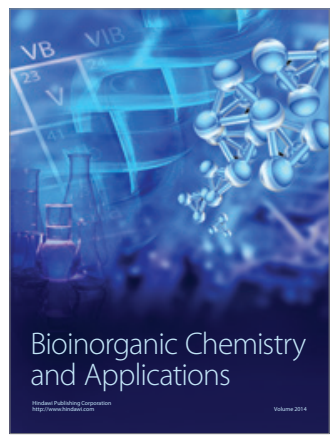

Inorganic Chemistry
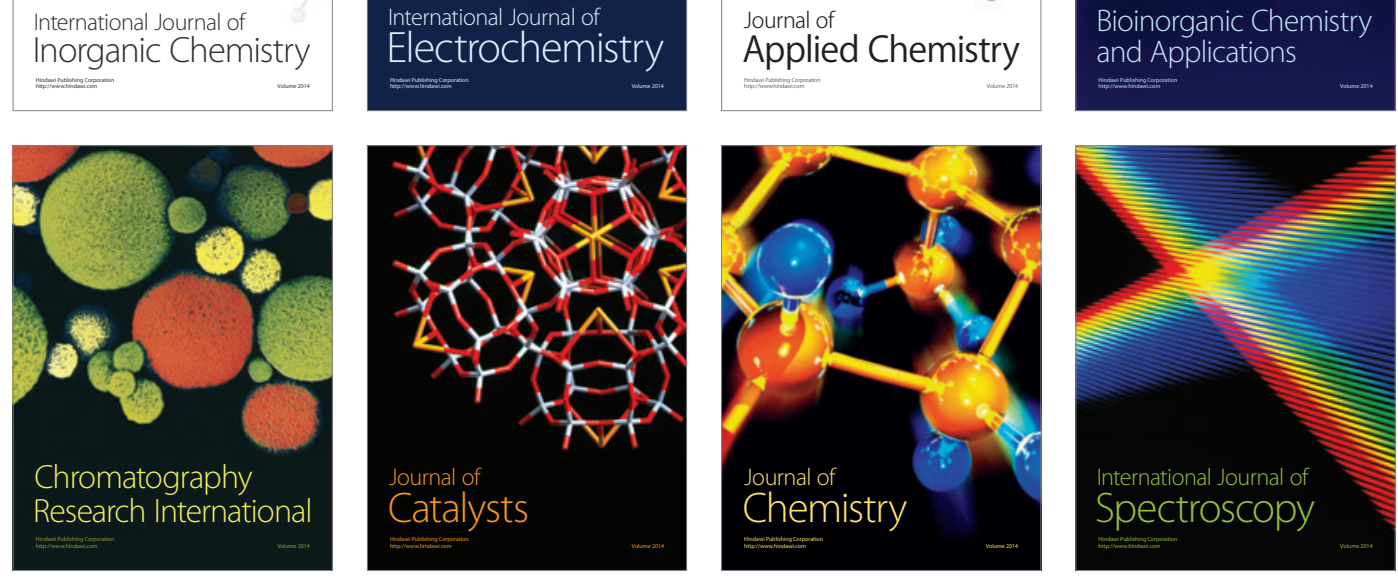